\title{
Exploring the Unknown: Electrophysiological and Behavioural Measures of Visuospatial Learning
}

\author{
Brendan Quinlivan \\ Trinity College Dublin \\ John Butler \\ Technological University Dublin, john.s.butler@tudublin.ie \\ Abdur R. Ridwan \\ Trinity College Dublin
}

See next page for additional authors

Follow this and additional works at: https://arrow.tudublin.ie/scschmatart

Part of the Mathematics Commons, and the Neuroscience and Neurobiology Commons

\section{Recommended Citation \\ Quinlivan, B. et al. (2016). Exploring the unknown: electrophysiological and behavioural measures of visuospatial learning. European Journal of Neuroscience Volume 43, Issue 9, pages 1128-1136. \\ doi:10.1111/ejn.13195}

This Article is brought to you for free and open access by the School of Mathematics at ARROW@TU Dublin. It has been accepted for inclusion in Articles by an authorized administrator of ARROW@TU Dublin. For more information, please contact arrow.admin@tudublin.ie, aisling.coyne@tudublin.ie,gerard.connolly@tudublin.ie.

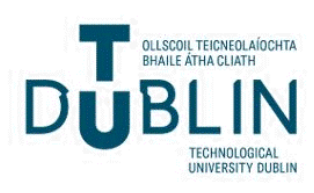




\section{Authors}

Brendan Quinlivan, John Butler, Abdur R. Ridwan, Ines Beiser, Laura Williams, Eavan McGovern, Sean O'Riordan, Michael Hutchinson, and Richard B. Reilly 
Received Date : 02-Oct-2015

Revised Date : 26-Jan-2016

Accepted Date : 27-Jan-2016

Article type : Research Report

Research Report: European Journal of Neuroscience

\title{
Exploring the unknown: Electrophysiological and behavioural measures of visuospatial learning
}

\author{
Brendan Quinlivan ${ }^{1,2 *}$, John S. Butler ${ }^{1,3}$, Abdur Raquib Ridwan ${ }^{1,2}$, Ines Beiser, ${ }^{5,6}$, Laura \\ Williams ${ }^{5,6}$, Eavan McGovern ${ }^{5,6}$, Sean O'Riordan ${ }^{5,6}$ Michael Hutchinson $^{5,6}$, Richard B. Reilly ${ }^{1,}$ \\ $2,4 *$
}

1: Trinity Centre for Bioengineering, Trinity College, Dublin, Ireland

2: School of Engineering, Trinity College, Dublin, Ireland

3: School of Mathematical Sciences, Dublin Institute of Technology, Kevin St, Dublin

4: School of Medicine, Trinity College, Dublin, Ireland,

5: Department of Neurology, St. Vincent's University Hospital, Dublin, Ireland

6: University College Dublin, Dublin, Ireland

* Corresponding author

email: quinlivb@tcd.ie, reillyri@tcd.ie

Running Title: Exploring the Unknown

Keywords - P3, P3b, alpha, visual learning, visuospatial memory

This article has been accepted for publication and undergone full peer review but has not been through the copyediting, typesetting, pagination and proofreading process, which may lead to differences between this version and the Version of Record. Please cite this article as doi: 10.1111/ejn.13195

This article is protected by copyright. All rights reserved. 
Abstract. Visuospatial memory describes our ability to temporarily store and manipulate visual and spatial information and is employed for a wide variety of complex cognitive tasks. Here a visuospatial learning task requiring fine motor control is employed to investigate visuospatial learning in a group of typically developing adults. Electrophysiological and behavioural data is collected during a target location task under two experimental conditions, Target Learning and Target Cued. Movement times are employed as a behavioural metric of performance while dynamic P3b amplitudes and power in the alpha band (approximately $10 \mathrm{~Hz}$ ) are explored as electrophysiological metrics during visuospatial learning. Results demonstrate that task performance, as measured by movement time, is highly correlated with P3b amplitude and alpha power at a consecutive trial level (trials 1 30). The current set of results, in conjunction with the existing literature, suggests that changes in P3b amplitude and alpha power could correspond to different aspects of the learning process. Here it is hypothesized that changes in P3b correspond to a diminishing inter-stimulus-interval and reduced stimulus relevance while the corresponding changes in alpha power represent an automation of response as habituation occurs in participants. The novel analysis presented in the current study demonstrates how gradual electrophysiological changes can be tracked during the visuospatial learning process under the current paradigm.

\section{Introduction}

Visuospatial memory is responsible for storing information about our environment and the spatial orientation of objects in that environment. Abnormal visuospatial processing has been observed in several clinical disorders including Autistic Spectrum Disorders (McGrath et al., 2012, McGrath et al., 2013), Parkinson's disease (Nantel et al., 2012, Leek et al., 2014) and Schizophrenia (White et al., 2011, Bourque et al., 2013). The widespread nature of abnormal visuospatial processing creates a need for objective methods of assessment. The task (as adapted from Stafford et al. (Stafford et al.,

This article is protected by copyright. All rights reserved. 
2012)) and novel analysis presented in the current study may have utility for the investigation of visuospatial processing for these clinical cohorts.

In a recent study Bednark et al. demonstrated a relationship between visuospatial learning and P3b amplitude (Bednark et al., 2013). Bednark investigated visuospatial learning by comparing behavioural movement times (MT) and electroencephalogram (EEG) components acquired during a target location task consisting of 30 repetitions (Bednark et al., 2013). In that study trials 1-15 were grouped together, as were trials 16-30. In this fashion Bednark et al. were able to show a relationship between P3b amplitude and MT across the first and second half of the task. The current study explores this relationship in greater detail by analysing behavioural and electrophysiological data at a consecutive trial level.

The P3b is a subcomponent of the P300 event related potential (ERP); it is a positive component usually peaking in the time window of $300-500$ ms post stimulus. The $\mathrm{P} 3 \mathrm{~b}$ is most commonly observed in tasks that require an aspect of performance monitoring and where stimuli are relevant to task performance (Polich, 2007, Courchesne et al., 1975). It has been observed that the amplitude of the P3b is modulated by factors such as stimulus relevance, stimulus occurrence probability (Duncan-Johnson and Donchin, 1977), stimulus complexity (Isreal et al., 1980, Johnson, 1986) and inter stimulus intervals (Gonsalvez and Polich, 2002, Steiner et al., 2014, Croft et al., 2003, Steiner et al., 2013, Polich, 2003). While there have been several studies that suggest a link between the P3b component and various types of learning (Sailer et al., 2010, Bednark et al., 2013, Lindin et al., 2004, Batterink et al., 2015, Jongsma et al., 2006), it is a small subsection of these that explicitly link the P3b to visuospatial learning (Bednark et al., 2013).

Changes of alpha oscillations $(8-12 \mathrm{~Hz})$ have been strongly linked to the cognitive state of participants. Power in the alpha band over occipital regions is generally observed to decrease during tasks requiring greater effort and attentional loads (Bollimunta et al., 2008, Ergenoglu et al., 2004, Snyder and Foxe, 2010). Increases in alpha power have been associated with decreased attentional

This article is protected by copyright. All rights reserved. 
demand and with improvements in learning based tasks (Bays et al., 2015, Dockree et al., 2007). One interpretation of these characteristics is that increases in alpha power during repetitive tasks represent an aspect of automation of response from participants: As responses become automatic, less attention is required which in turn leads to increased alpha power (Jensen and Mazaheri, 2010, Bays et al., 2015). However, alpha power is also known to increase with time spent of repetitive tasks and is often accompanied by reduced performance (Foxe et al., 2012), reflecting a decrease in concentration by participants. This confound implies that for increased alpha power to represent an increase in automation, behavioural performance must also remain consistent. This interpretation is only appropriate in tasks where habituation of response is possible, such as the current paradigm.

This study confirms and extends the findings of Bednark and colleagues. In addition to investigating the P3b response, a novel analysis examines changes in alpha power during the learning process. P3b amplitudes and alpha power values were analysed at a consecutive trial level to study the dynamic time course of visuospatial learning across 30 consecutive trials. Correlation analysis was carried out to compare electrophysiological and behavioural metrics. Finally correlation analysis was carried out at single participant level to test the reproducibility of the proposed electrophysiological metrics. We hypothesize that the relationship between P3b amplitude and task performance is not an indication that P3b amplitude is reflecting visuospatial learning directly but may be driven by other well-documented properties of the P3b component.

\section{Materials and Methods}

\subsection{Participants/Ethics}

Eleven healthy adults participated in this study $(25.3 \pm 2.6$ years, mean \pm SD). All participants were right-handed as revealed by self-report and had normal or corrected to normal vision. Only male participants were included to account for gender imbalances in visuospatial processing (Roalf et al., 2006). In accordance with the declaration of Helsinki, all participants gave their written informed

This article is protected by copyright. All rights reserved. 
consent to the study, which was approved by the Faculty Ethics Committee of the Faculty of Health Sciences at Trinity College Dublin.

\subsection{The Task}

The task used in the current study was adapted from previous work by Stafford et al. and Bednark et al. (Stafford et al., 2012, Bednark et al., 2013). Participants were seated in a comfortable chair, 70 $\mathrm{cm}$ from a computer monitor in a dark and quiet room where they were asked to complete a behavioural experiment. Participants were presented with a black screen containing a white cursor $\left(0.8^{\circ}\right.$ diameter, visual degrees), a grey cross hair and in some conditions a grey annulus, as shown in Figure 1. Movement of the cursor within a bounded, square search space ( $10.8^{\circ}$ width) was controlled using a Kensington Orbit Optical Trackball.

Prior to the experiment participants were informed that their aim was to move the cursor into a circular target location $\left(3.2^{\circ}\right.$ diameter) in the fastest and most accurate manner possible. Participants completed a shortened practice session to help familiarize them with the paradigm. Providing detailed instructions and practice sessions to the participants represents a departure from the protocol of Bednark et al. but was introduced to eliminate unwanted learning effects in the opening blocks. The practice session consisted of 10 trials of each experimental condition with a single target location for each of the 10 trials. At the beginning of each trial the cursor appeared at a new random starting position inside the search space. To complete a single trial, participants were required to manoeuvre the cursor into the target location. When the cursor entered the target location a green circular stimulus $\left(1.1^{\circ}\right.$ diameter) was presented at the centre of the search space for $500 \mathrm{~ms}$, as shown in Figure 1. Each experimental block consisted of 30 trials. Within each block, the position of the target location remained consistent across all trials. At the beginning of each block a new target location was randomly generated. The target location could be generated at any point inside the search space providing it was never placed at the centre of the search space or directly

This article is protected by copyright. All rights reserved. 
adjacent to a bounding wall. This stipulation helped to reduce learning of target location in relation to visible landmarks and encourage visuospatial learning of the search space.

Participants completed this task under two conditions, Target Learning (TL) and Target Cued (TC). In the TL condition the position of the target location was hidden and participants were expected to learn the new location over the course of each block. In the TC condition the target location was highlighted by a grey annulus. The TC condition was designed as a control condition where visuospatial learning would not occur but a motor response would still be present. Participants completed 10 experimental blocks for each condition in alternating order.

\subsection{Data Acquisition}

Continuous EEG data, sampled at $512 \mathrm{~Hz}$, was collected from 64 scalp and 8 external active sintered $\mathrm{Ag}-\mathrm{AgCl}$ electrodes using a BioSemi high impedance recording system. Stimuli were generated and presented using Neurobehavioral Systems' Presentation software. Triggers were recorded at stimulus onset for offline EEG analysis. The international 10-20 electrode layout system was employed for recordings.

\subsection{Data Analysis}

Movement time (MT) was defined as the time interval from the beginning of each trial to the point that the participant manoeuvred the cursor into the target location. Average MTs were calculated for all participants and group average MTs were calculated across participants to allow for comparison with group average ERP data.

\subsubsection{ERP Analysis}

EEG data were analysed using custom MATLAB R2013b scripts and EEGLAB (Delorme and Makeig, 2004). Data were first de-trended by removing the line of best fit from each channel to correct for signal drift. The data were then filtered using a $4^{\text {th }}$ order band-pass Butterworth filter with a pass band of $0.1-40 \mathrm{~Hz}$. An external electrode on the nasion was employed to reject trials that contained

This article is protected by copyright. All rights reserved. 
eye blinks; any channel that contained a voltage greater than $\pm 80 \mu \mathrm{V}$ was also rejected. Trials that consisted of more than $10 \%$ bad channels were excluded from further analysis. In trials where the number of bad channels was fewer than $10 \%$, those bad channels were interpolated using four nearest "good" neighbours, as described in (Butler et al., 2011). Average reference was employed during analysis by re-referencing the data to the average of all channels (Michel et al., 2004, Nunez and Srinivasan, 2006); a 80 ms pre stimulus window was used as the baseline period. Average ERPs to feedback stimulus onset were calculated for a $600 \mathrm{~ms}$ window for each trial. Individual ERPs were calculated across all blocks for the TL and TC experimental conditions for each trial. Group average ERPs were calculated across all participants. P3b amplitudes were calculated for each trial at group mean level by taking the mean value of the ERP waveform over the time interval 310-390 ms poststimulus (Leue and Beauducel, 2015). This time window was selected to include the peak of the P3b component across all trials. This analysis was carried out over 9 central electrodes of interest (FC1, $\mathrm{FC} 2, \mathrm{FCz}, \mathrm{CP} 1, \mathrm{CP} 2, \mathrm{CPz}, \mathrm{C} 1, \mathrm{C2}, \mathrm{Cz}$ ) and the mean of these was taken as the $\mathrm{P} 3 \mathrm{~b}$ amplitude for a given trial.

\subsubsection{Spectral Analysis}

Power in the alpha band was estimated using a 500 ms time window prior to target location and the presentation of the feedback stimulus. This time window was selected to provide electrophysiological data during participant response. Given that alpha oscillations are not stimulus locked it was necessary to calculate alpha power at a single trial level. To calculate the power in the $8-12 \mathrm{~Hz}$ spectral band the Fourier Transform of the $500 \mathrm{~ms}$ window was calculated and the average power across the specified band was estimated. The alpha power of a single trial was calculated as the mean power in the $8-12 \mathrm{~Hz}$ frequency band. Electrodes of interest (CP3, CP1, CPz, P5, P3, P1, $\mathrm{Pz}, \mathrm{P} 2, \mathrm{PO}, \mathrm{POz}, \mathrm{O} 1, \mathrm{Oz}$ ) were used to calculate alpha power, where the topography showed alpha power to be most prominent. Group average alpha power was then calculated by generating the mean across blocks and participants.

This article is protected by copyright. All rights reserved. 


\subsection{Statistical Analysis}

\subsubsection{Variance Analysis}

MTs were submitted to a 2-way ANOVA to compare the mean differences between the TL and TC conditions. The independent variables used for this analysis were condition (level $=2, T L$ and TC) and trial (level = 30, trials $1-30$ ); the dependent variable was MT. In follow up analysis, MTs from the TL and TC condition were submitted to a 1-way ANOVA to determine if significant differences existed due to trial number. The independent variable for this test was trial (level $=30$, trials $1-30$ ) and the dependent variable was MT. This analysis was carried out to explore how the performance of participants varied over trials and across conditions. P3b amplitude data and alpha power data were submitted to identical 2-way and 1-way ANOVA analysis. The aim of this analysis was to determine the significance of the observed changes in P3b amplitude and power in the alpha band across trials and condition. A Greenhouse-Geisser correction was applied to all analysis of variance carried out here.

Due to the unique exploratory nature of trial 1 in the TL condition, it was hypothesised that results from this trial could strongly influence the ANOVA analysis. For this reason all ANOVAs were carried out twice, with and without inclusion of trial 1.

\subsubsection{Correlation Analysis}

Correlation analysis was carried out on P3b and MT data at the group mean level to explore the relationship between P3b amplitude and task performance in both the TL and TC experimental conditions across the 30 trials of the block. Alpha power was also compared against MT to determine the statistical relationship between these metrics. Finally, correlation analysis of alpha power and P3b amplitude data was carried out to determine if these distinct electrophysiological parameters were statistically related. Correlations were calculated using the grand average values to test for correlation over the 30 trials of the experimental block.

This article is protected by copyright. All rights reserved. 
As with the ANOVA, all correlation tests were carried out twice, once with inclusion of trial 1 and once without. A significance level of 0.015 was selected to account for multiple comparisons of the MT variable (Bonferroni correction).

In order to test the reproducibility of the results at single participant level, single participant MT, P3b amplitude and alpha rhythm power averages were calculated and submitted to the same correlation analysis as outlined for the grand average data above. Single participant correlations were calculated using the average across blocks for all trials. All reproducibility analysis was carried out with exclusion of trial 1 . As with the group mean correlations, a significance level of 0.015 was selected to account for multiple comparisons.

\section{Results}

\subsection{Behavioural}

The behavioural measure of performance employed in this study was MT, the time taken for the participant to move the cursor into the target location in each trial. Figure 2(a) shows the group average MTs for both the TL and TC tasks. Figure 2(b) shows group average MTs with exclusion of trial 1. A steep increase in performance is observed over the first 5 trials of the TL task; MTs remain consistent over the remaining 25 trials. MTs remain consistent across the TL task with no obvious learning effect. A 2 (Condition: TL, TC) $\times 30$ (trial: 1 to 30) repeated measures ANOVA was conducted on MTs and demonstrated a main effect of condition $(F(1,10)=75.7, p<0.001)$, a main effect of trial $(F(1.3,12.9)=51.8, p<0.001)$ and an interaction effect $(F(1.3,13.1)=49.8, p<0.001)$. This main effect of condition shows MTs are slower in the TL condition. Planned comparisons demonstrated significant differences between trials for the $T L$ condition $(F(1.3,12.9)=51.2, p<0.001)$ but not for the TC condition $(F(3.7,37.2)=1.8, p=0.15)$. Exclusion of trial 1 from the MT ANOVA analysis did not alter the significance of the results; see Table 1 . These results demonstrate that participants

This article is protected by copyright. All rights reserved. 
become progressively faster at the task in the TL condition over trials but not in the TC condition. A summary of repeated measures ANOVA and planned comparison analysis is displayed in Table 1.

\subsection{Electrophysiological}

\subsection{1. $\quad$ ERP Results}

Figure 3(a) depicts the group average ERPs for the TL condition and Figure 3(b) shows the corresponding ERPs for the TC condition. There was no visible change in P3b amplitude after the $5^{\text {th }}$ trial in either condition, therefore trials 6-30 are plotted as a mean curve with standard error displayed. The topographical distribution of the P3b component for both experimental conditions was calculated over an $80 \mathrm{~ms}$ window from 310-390 ms post stimulus onset. Group average topographical distributions for the first 5 trials of each condition are displayed, Figure 3(c). P3b amplitude was calculated for each of the consecutive 30 trials for the TL and TC condition, as described in the materials and methods section. These P3b amplitudes and corresponding standard errors were plotted for each condition to illustrate the observed change in P3b amplitude over the experimental block, as shown in Figure 3(d). The 2 (Condition: TL, TC) $\times 30$ (trial: 1 to 30) repeated measures ANOVA on the P3b amplitudes showed a significant effect of condition $(F(1,10)=35.5, p<$ $0.001)$, a significant effect of trial $(F(7.2,72.1)=6.1, p<0.001)$ and a significant interaction of condition by trial $(F(6.1,61.2)=7.7, p<0.001)$. This main effect of condition shows that P3b amplitude is larger in the TL condition. Planned within condition one-way repeated measures comparisons demonstrated significant differences between trials for the $T L$ condition $(F(6.4,63.8)=$ $12.5, p<0.001)$ but not for the TC condition $(F(5.9,59.4)=0.7, p=0.63)$. Exclusion of trial 1 from the P3b amplitude ANOVA analysis did not alter the significance of the results; see Table 1. This analysis demonstrates that P3b amplitude decreases over trials in the TL condition but remains consistently non-existent in the TC condition.

This article is protected by copyright. All rights reserved. 


\subsubsection{Spectral Results}

In both the TL and TC conditions, an increasing trend in alpha power is observed over the course of the experimental block, as shown in Figure 4(a, b). A two (Condition: TL \& TC) $\times 30$ (trial: 1 to 30) repeated measures ANOVA revealed a significant effect of condition $(F(1,10)=5.1, p<0.05)$, a significant effect of trial $(F(4.7,47.2)=7.5, p<0.001)$ with no interaction effect $(F(5.5,54.6)=1.5, p$ $=0.187)$. This main effect of condition represents that fact that alpha power is larger in the TC condition than in the TL condition, this is likely due to the lower attentional level required in the cued condition. This result may be influenced by the fact that alpha power appears to increase to its peak value earlier in the TC condition, as can be observed in Figure 4, however, this is not statistically supported as no interaction of condition and trial was present. Planned one-way ANOVA comparisons showed a significant effect of trial in both the TL condition $(F(5.1,51.3)=4.3, p$ $<0.05)$ and TC condition $(F(4.8,47.8)=5.6 p<0.001)$. These results indicate that alpha power increases with trial number in both the TL and TC conditions. Exclusion of trial 1 from the alpha power ANOVA analysis did not alter the significance of these results; see Table 1.

\subsubsection{Correlation}

Correlation analysis of P3b amplitude and MT showed a significant relationship in the TL condition (R $=0.85, p<0.001)$ with no significant relationship in the TC condition $(p=0.828)$. The correlation in the TL condition maintained with exclusion of trial $1(R=0.95, p<0.001)$ while, again, no relationship was observed in the TC condition $(p=0.14)$. The positive correlation of P3b and MT in the TL task indicates that the amplitude of the P3b decreases with the reduction in MT. This relationship is not observed in the TC condition.

Alpha power was tested against MT to determine if it had any relationship with participant performance during the task. A relationship was observed between alpha power and MT for the TL experimental condition $(R=-0.47, p<0.015)$ while no significant relationship was seen in the TC condition $(p=0.14)$. Exclusion of the first trial did not alter the significance of the results; see Table

This article is protected by copyright. All rights reserved. 
2. This negative correlation in the TL condition indicates that alpha power increases with improvement in task performance as measured by decreasing MTs.

To probe the relationship between $\mathrm{P} 3 \mathrm{~b}$ amplitude and alpha power, a correlation analysis was carried out. At group mean level, the correlation analysis showed a significant relationship between these distinct electrophysiological parameters $(R=-0.67, p<0.001)$ in the TL experimental condition. No relationship was observed in the TC condition $(p=0.15)$. Exclusion of the first trial did not alter the significance of the results. A summary of these results are displayed in Table 2 and illustrated in Figure 5.

To test the reproducibility of P3b amplitude and alpha power, correlations were calculated at single participant level. The corresponding $p$-values are summarized in Table 3. A significant relationship was observed between MT and P3b amplitude in 8 of the 11 participants in the TL condition, the remaining participants showed no relationship between $\mathrm{MT}$ and P3b amplitude. In the TC condition, no significant relationship was observed between MT and P3b amplitude. This result shows that the relationship between $\mathrm{P} 3 \mathrm{~b}$ amplitude and $\mathrm{MT}$ is highly reproducible at single participant level. A significant relationship between MT and alpha power was observed in 6 participants in the TL condition. In the TC condition there was only one significant relationship across participants. Finally the reproducibility of the relationship between P3b amplitude and alpha power was tested. It was observed in the TL condition that this result was significant in 2 participants only. In the TC condition one significant relationship was observed. This result would suggest that the relationship between P3b and alpha is not highly reproducible at the single participant level.

\section{Discussion}

The current study is an investigation of electrophysiological and behavioural measures collected during visuospatial learning in a motor search task. Given our results and the existing literature, it is hypothesised that the observed changes in task performance, as measured by movement time (MT),

This article is protected by copyright. All rights reserved. 
are influenced though a combination of visuospatial learning and automation of response. Furthermore, it is suggested that the observed changes in P3b amplitude primarily reflect a decreasing MT, rather than visuospatial learning directly.

\subsection{Behavioural}

MT was employed as a behavioural metric of task performance (Stafford et al., 2012, Bednark et al., 2013, Thirkettle et al., 2013). Results show a significant decrease in MT across the first 5 trials in the TL condition, no such change is visible in the TC. In the TL condition MTs decrease to a low plateau, although they still remain slower than that of the TC condition. It is hypothesized that the observed change in MT in the TL condition primarily represents the process of visuospatial learning, however, there may also be a secondary effect on performance due to automation of response. It is possible that the use of more sensitive metrics would allow these effects to be distinguished.

\subsection{Electrophysiological}

The P3b has long been associated with the process of decision making (Hillyard et al., 1971, Sutton et al., 1965). Recent studies continue to demonstrate how our understanding of these components can continue to evolve through the development of specific and well-designed experimental protocols (O'Connell et al., 2012, Kelly and O'Connell, 2013, Twomey et al., 2015). Bednark et al. demonstrated that both MT and the amplitude of the P3b component decreased between the first and second half of this 30 trial experiment (Bednark et al., 2013). In the current study P3b amplitude was analysed at consecutive trial level across all 30 trials. This novel analytic approach facilitated a deeper investigation into the relationship between visuospatial learning and P3b amplitude. Our results reveal a strong relationship between P3b amplitude and MT in the TL condition, which is highly consistent with the results presented by Bednark and colleagues. An important property of the $\mathrm{P} 3 \mathrm{~b}$ component is that its amplitude is modulated by inter-stimulus intervals (ISI) (Gonsalvez and Polich, 2002, Steiner et al., 2014, Croft et al., 2003, Steiner et al., 2013,

This article is protected by copyright. All rights reserved. 
Polich, 2003). Given that MT directly equates to ISI in this task, it is possible that the observed decrease in P3b amplitude is an effect of the decreasing MT (and ISI). If the change in P3b amplitude is an effect of ISI then this change could only be considered a secondary effect of visuospatial learning rather than a direct measure. Bednark et al. addressed this concern through inclusion of a third condition in which cursor movement did not elicit a stimulus but ISI was matched to the TL condition, i.e. movement $\neq$ outcome. In this condition, no relationship was observed between P3b amplitude and MT. This result could suggest that the change in P3b is not a factor of ISI, however, it could also result from lowered participant engagement during a task where one's responses are meaningless and stimulus relevance has therefore been removed.

P3b amplitude is also modulated by stimulus relevance for a wide range of visual and auditory tasks (Sawaki and Katayama, 2006, Briggs and Martin, 2009). This property of the P3b may also have a role in the observed correlation. It is plausible that stimulus relevance is higher during the early stages of learning and decreases as the target location is learned and feedback is no longer required. This decrease in stimulus relevance during visuospatial learning could explain why a decrease in P3b amplitude is observed over the early trials of the TL condition. This mechanism would also explain why the TC condition failed to elicit a P3b: As the participant always knows the target location, the feedback stimulus is not relevant to task completion and would therefore not be expected to produce a P3b response.

Whether the change in P3b amplitude in the TL condition is a factor of ISI, decreasing stimulus relevance or a combination of the two, it has utility as an electrophysiological metric of task performance rather than a direct measure of visuospatial learning. While it is possible that the P3b component is reflecting visuospatial learning independently of ISI in the current task, the strength of the correlation observed here and the extensive literature on this point (Gonsalvez and Polich, 2002, Steiner et al., 2014, Croft et al., 2003, Steiner et al., 2013, Polich, 2003) reduce the plausibility of this hypothesis.

This article is protected by copyright. All rights reserved. 
In both the TL and TC condition of this study a significant increase in alpha power was observed across trials. Increases in alpha power during repetitive visual tasks can be explained by two distinct mechanisms. Some studies suggest that increases in alpha power reflect a level in automation during repetitive tasks where it is possible for the participant to learn the required response (Jensen and Mazaheri, 2010, Bays et al., 2015). In such studies, increases in alpha power have been observed to correlate with improvements in performance. On the other hand, when habituation of response is not possible, alpha power has been observed to increase with time-on-task and has been seen to correlate with reduced performance (Foxe et al., 2012). In both cases, it seems that alpha power tends to increase as attention levels decrease. As habituation of response is possible in the current paradigm and performance is not observed to decrease with increases is alpha, it is plausible that the increase observed here represents a level of automation of response and a reduced attention load over the experimental block. Alpha power has also been observed to undergo event related desynchronization in the time window of $400-700$ ms post stimulus presentation (Wright et al., 2015, Makin et al., 2014, Klimesch et al., 2007). Given than the 500 ms pre stimulus time window employed in the current study will overlap with this $400-700 \mathrm{~ms}$ time window from the previous trial in some cases, it is possible that the observed increases in alpha power could be impacted by a reduced level of event related desynchronization.

The fact that this result is present in both experimental conditions would suggest that this automation occurs in both the TL and TC condition. For this hypothesis to be accurate, differences observed between the TL and TC conditions for MT and P3b amplitude data must be explained by a process other than automation of response. This agrees with our previous hypothesis that those changes were a result of improved task performance, which we suggested are primarily driven by visuospatial learning with automation of response as a smaller secondary factor. One limitation to this theory is that no correlation is observed between alpha power and MT in the TC condition, as would be expected under the given hypothesis. This may be explained by a lack of sensitivity of the performance metric MT.

This article is protected by copyright. All rights reserved. 


\subsection{Correlation analysis}

The correlation analysis for the TL condition revealed a significant relationship between alpha power and MT and between P3b amplitude and MT. No such relationship was observed in the TC condition. These results support the view that MT reflects an overall measurement of performance, which is affected by changes in visuospatial learning and automation of response. Furthermore, the results of the correlation analysis reveal that this change in alpha power is strongly related to the observed change in P3b amplitude in the TL condition at the group mean level. Given the hypothesis that the changes in P3b are driven by changes in $\mathrm{MT}$, this correlation likely reflects the alpha MT correlation rather than a separate relationship between $\mathrm{P} 3 \mathrm{~b}$ and alpha power. The results of the single participant correlation analysis reveal that the relationships between P3b amplitude and MT and alpha power and MT are both highly reproducible. The relationship between P3b and alpha was not robust at this level, supporting the view that the correlation at group level was not meaningful. These results support the group average correlations and suggest that the current paradigm and proposed metrics have utility for research at a single participant level.

\subsection{Concluding Remarks}

The task presented here has many qualities that make it suitable for the study of visuospatial learning; it is repeatable without a learning effect, it provides an objective measure of performance, it is intuitive and engaging for participants and it is scalable in difficulty (Stafford et al., 2012). These qualities make it suitable for deployment in movement disorders studies such as Parkinson's disease or dystonia where participants have varying levels of motor control. The novel analysis presented here highlights how P3b amplitudes and alpha power can be assessed at a consecutive trial level and demonstrates the versatility of such tasks in visuospatial learning research.

This article is protected by copyright. All rights reserved. 
In the current study, it has been demonstrated that performance in this visuospatial learning task, as measured by movement time, is highly correlated with both alpha power and P3b amplitude at a consecutive trial level. It is hypothesised here that the relationship between MT and P3b amplitude is driven by factors such as ISI and stimulus novelty rather than being a direct reflection of visuospatial learning. The hypothesis that alpha power could reflect an increasing level of automation in the given task is supported by the correlation between alpha power and MT in the TL condition but is hindered by the lack of correlation in the TC condition. It is possible that the use of a more sensitive behavioural measure would allow for more definitive conclusions on this point but further research is required to fully address this question. The analysis carried out here has shown that the data collected in this paradigm is robust enough to be analysed at a consecutive trial level. This novel analytic approach has allowed for a deeper understanding of the relationships between $\mathrm{MT}, \mathrm{P} 3 \mathrm{~b}$ amplitudes and alpha power and has informed the hypotheses presented in the current work. Another important implication of this work is that the analysis presented by Bednark et al. (Bednark et al., 2013) could likely be carried out with less data, allowing for shorter recording sessions in clinical populations. This work has also demonstrated that this analysis is highly reproducible at a single participant level. This gives the current paradigm further utility in clinical populations where inter-participant variability may be inflated, and group mean analysis may not be appropriate.

\section{Acknowledgements}

This work was primarily supported by a grant from the Health Research Board of Ireland (CSA 20125). The authors would like to thank the participants who donated their time. The authors declare no competing financial interests that would bias the results reported here. Finally the authors wish to acknowledge Prof. Rolf Verleger for his constructive and insightful input to the current manuscript.

This article is protected by copyright. All rights reserved. 


\section{Abbreviations}

- EEG: Electroencephalogram

- ERP: Event-related potential

- ISI: Inter stimulus interval (ISI)

- MT: Movement time

- TC: Target Cued

- TL: Target Learning

\section{References}

BATTERINK, L. J., REBER, P. J., NEVILLE, H. J. \& PALLER, K. A. 2015. Implicit and explicit contributions to statistical learning. J Mem Lang, 83, 62-78.

BAYS, B. C., VISSCHER, K. M., LE DANTEC, C. C. \& SEITZ, A. R. 2015. Alpha-band EEG activity in perceptual learning. J Vis, 15, 7.

BEDNARK, J. G., REYNOLDS, J. N., STAFFORD, T., REDGRAVE, P. \& FRANZ, E. A. 2013. Creating a movement heuristic for voluntary action: electrophysiological correlates of movementoutcome learning. Cortex, 49, 771-80.

BOLLIMUNTA, A., CHEN, Y., SCHROEDER, C. E. \& DING, M. 2008. Neuronal mechanisms of cortical alpha oscillations in awake-behaving macaques. J Neurosci, 28, 9976-88.

BOURQUE, J., LAKIS, N., CHAMPAGNE, J., STIP, E., LALONDE, P., LIPP, O. \& MENDREK, A. 2013. Clozapine and visuospatial processing in treatment-resistant schizophrenia. Cogn Neuropsychiatry, 18, 615-30.

BRIGGS, K. E. \& MARTIN, F. H. 2009. Affective picture processing and motivational relevance: arousal and valence effects on ERPs in an oddball task. Int J Psychophysiol, 72, 299-306.

BUTLER, J. S., MOLHOLM, S., FIEBELKORN, I. C., MERCIER, M. R., SCHWARTZ, T. H. \& FOXE, J. J. 2011. Common or redundant neural circuits for duration processing across audition and touch. $J$ Neurosci, 31, 3400-6.

COURCHESNE, E., HILLYARD, S. A. \& GALAMBOS, R. 1975. Stimulus novelty, task relevance and the visual evoked potential in man. Electroencephalogr Clin Neurophysiol, 39, 131-43.

CROFT, R. J., GONSALVEZ, C. J., GABRIEL, C. \& BARRY, R. J. 2003. Target-to-target interval versus probability effects on P300 in one- and two-tone tasks. Psychophysiology, 40, 322-8.

DELORME, A. \& MAKEIG, S. 2004. EEGLAB: an open source toolbox for analysis of single-trial EEG dynamics including independent component analysis. J Neurosci Methods, 134, 9-21.

DOCKREE, P. M., KELLY, S. P., FOXE, J. J., REILLY, R. B. \& ROBERTSON, I. H. 2007. Optimal sustained attention is linked to the spectral content of background EEG activity: greater ongoing tonic alpha (approximately $10 \mathrm{~Hz}$ ) power supports successful phasic goal activation. Eur J Neurosci, 25, 900-7.

DUNCAN-JOHNSON, C. C. \& DONCHIN, E. 1977. On quantifying surprise: the variation of eventrelated potentials with subjective probability. Psychophysiology, 14, 456-67.

ERGENOGLU, T., DEMIRALP, T., BAYRAKTAROGLU, Z., ERGEN, M., BEYDAGI, H. \& URESIN, Y. 2004. Alpha rhythm of the EEG modulates visual detection performance in humans. Brain Res Cogn Brain Res, 20, 376-83.

FOXE, J. J., MORIE, K. P., LAUD, P. J., ROWSON, M. J., DE BRUIN, E. A. \& KELLY, S. P. 2012. Assessing the effects of caffeine and theanine on the maintenance of vigilance during a sustained attention task. Neuropharmacology, 62, 2320-7.

GONSALVEZ, C. L. \& POLICH, J. 2002. P300 amplitude is determined by target-to-target interval. Psychophysiology, 39, 388-96.

This article is protected by copyright. All rights reserved. 
HILLYARD, S. A., SQUIRES, K. C., BAUER, J. W. \& LINDSAY, P. H. 1971. Evoked potential correlates of auditory signal detection. Science, 172, 1357-60.

ISREAL, J. B., CHESNEY, G. L., WICKENS, C. D. \& DONCHIN, E. 1980. P300 and tracking difficulty: evidence for multiple resources in dual-task performance. Psychophysiology, 17, 259-73.

JENSEN, O. \& MAZAHERI, A. 2010. Shaping functional architecture by oscillatory alpha activity: gating by inhibition. Front Hum Neurosci, 4, 186.

JOHNSON, R., JR. 1986. A triarchic model of P300 amplitude. Psychophysiology, 23, 367-84.

JONGSMA, M. L., EICHELE, T., VAN RIJN, C. M., COENEN, A. M., HUGDAHL, K., NORDBY, H. \& QUIROGA, R. Q. 2006. Tracking pattern learning with single-trial event-related potentials. Clin Neurophysiol, 117, 1957-73.

KELLY, S. P. \& O'CONNELL, R. G. 2013. Internal and external influences on the rate of sensory evidence accumulation in the human brain. J Neurosci, 33, 19434-41.

KLIMESCH, W., SAUSENG, P. \& HANSLMAYR, S. 2007. EEG alpha oscillations: the inhibition-timing hypothesis. Brain Res Rev, 53, 63-88.

LEEK, E. C., KERAI, J. H., JOHNSTON, S. J., HINDLE, J. V. \& BRACEWELL, R. M. 2014. Impaired visuospatial transformation but intact sequence processing in Parkinson disease. Cogn Behav Neurol, 27, 130-8.

LEUE, A. \& BEAUDUCEL, A. 2015. Effects of injustice sensitivity and sex on the P3 amplitude during deception. Biol Psychol, 109, 29-36.

LINDIN, M., ZURRON, M. \& DIAZ, F. 2004. Changes in P300 amplitude during an active standard auditory oddball task. Biol Psychol, 66, 153-67.

MAKIN, A. D., RAMPONE, G., WRIGHT, A., MARTINOVIC, J. \& BERTAMINI, M. 2014. Visual symmetry in objects and gaps. J Vis, 14, 12.

MCGRATH, J., JOHNSON, K., ECKER, C., O'HANLON, E., GILL, M., GALLAGHER, L. \& GARAVAN, H. 2012. Atypical visuospatial processing in autism: insights from functional connectivity analysis. Autism Res, 5, 314-30.

MCGRATH, J., JOHNSON, K., O'HANLON, E., GARAVAN, H., LEEMANS, A. \& GALLAGHER, L. 2013. Abnormal functional connectivity during visuospatial processing is associated with disrupted organisation of white matter in autism. Front Hum Neurosci, 7, 434.

MICHEL, C. M., MURRAY, M. M., LANTZ, G., GONZALEZ, S., SPINELLI, L. \& GRAVE DE PERALTA, R. 2004. EEG source imaging. Clin Neurophysiol, 115, 2195-222.

NANTEL, J., MCDONALD, J. C., TAN, S. \& BRONTE-STEWART, H. 2012. Deficits in visuospatial processing contribute to quantitative measures of freezing of gait in Parkinson's disease. Neuroscience, 221, 151-6.

NUNEZ, P. L. \& SRINIVASAN, R. 2006. Electric fields of the brain: the neurophysics of EEG, Oxford university press.

O'CONNELL, R. G., DOCKREE, P. M. \& KELLY, S. P. 2012. A supramodal accumulation-to-bound signal that determines perceptual decisions in humans. Nat Neurosci, 15, 1729-35.

POLICH, J. 2003. Theoretical overview of P3a and P3b, Detection of Change: Event-Related Potential and fMRI Findings, Kluwer Academic Press.

POLICH, J. 2007. Updating P300: an integrative theory of P3a and P3b. Clin Neurophysiol, 118, 212848.

ROALF, D., LOWERY, N. \& TURETSKY, B. I. 2006. Behavioral and physiological findings of gender differences in global-local visual processing. Brain Cogn, 60, 32-42.

SAILER, U., FISCHMEISTER, F. P. \& BAUER, H. 2010. Effects of learning on feedback-related brain potentials in a decision-making task. Brain Res, 1342, 85-93.

SAWAKI, R. \& KATAYAMA, J. 2006. Stimulus context determines whether non-target stimuli are processed as task-relevant or distractor information. Clin Neurophysiol, 117, 2532-9.

SNYDER, A. C. \& FOXE, J. J. 2010. Anticipatory attentional suppression of visual features indexed by oscillatory alpha-band power increases: a high-density electrical mapping study. J Neurosci, 30, 4024-32.

This article is protected by copyright. All rights reserved. 
STAFFORD, T., THIRKETTLE, M., WALTON, T., VAUTRELLE, N., HETHERINGTON, L., PORT, M., GURNEY, K. \& REDGRAVE, P. 2012. A novel task for the investigation of action acquisition. PLoS One, 7, e37749.

STEINER, G. Z., BARRY, R. J. \& GONSALVEZ, C. J. 2013. Can working memory predict target-to-target interval effects in the P300? Int J Psychophysiol, 89, 399-408.

STEINER, G. Z., BARRY, R. J. \& GONSALVEZ, C. J. 2014. Stimulus-to-matching-stimulus interval influences N1, P2, and P3b in an equiprobable Go/NoGo task. Int J Psychophysiol, 94, 59-68.

SUTTON, S., BRAREN, M., ZUBIN, J. \& JOHN, E. R. 1965. Evoked-potential correlates of stimulus uncertainty. Science, 150, 1187-8.

THIRKETTLE, M., WALTON, T., SHAH, A., GURNEY, K., REDGRAVE, P. \& STAFFORD, T. 2013. The path to learning: action acquisition is impaired when visual reinforcement signals must first access cortex. Behav Brain Res, 243, 267-72.

TWOMEY, D. M., MURPHY, P. R., KELLY, S. P. \& O'CONNELL, R. G. 2015. The classic P300 encodes a build-to-threshold decision variable. Eur J Neurosci, 42, 1636-43.

WHITE, T., HONGWANISHKUL, D. \& SCHMIDT, M. 2011. Increased anterior cingulate and temporal lobe activity during visuospatial working memory in children and adolescents with schizophrenia. Schizophr Res, 125, 118-28.

WRIGHT, D., MAKIN, A. D. \& BERTAMINI, M. 2015. Right-lateralized alpha desynchronization during regularity discrimination: hemispheric specialization or directed spatial attention? Psychophysiology, 52, 638-47.

Table 1. Summary of all repeated measures ANOVA and planned comparison analysis for movement times (MT), P3b amplitude and alpha power. F- and p-values are reported for all analysis carried out with inclusion of trial 1 and with exclusion of trial 1 (ex. 1). All main and interaction effects are reported, as are degrees of freedom (df). Greenhouse-Geisser corrected.

MT

\begin{tabular}{lllllllll} 
& & df & F & p & df & F ex. 1 & p ex. 1 \\
\hline 2-way ANOVA & Condition & 1,10 & 75.7 & $<0.001$ & 1,10 & 34.1 & $<0.001$ \\
& Trial & $1.3,12.9$ & 51.8 & $<0.001$ & $2.4,24.2$ & 11.2 & $<0.001$ \\
& & & & & & & & \\
& Cond. ${ }^{*}$ Trial & $1.3,13.1$ & 49.8 & $<0.001$ & $2.5,25.1$ & 10.7 & $<0.001$
\end{tabular}

Planned Comp.

$\begin{array}{llllllll}\text { (Trials) TC } & \text { Trials } & 3.7,37.2 & 1.8 & 0.15 & 7.7,76.6 & 1.3 & 0.117 \\ \text { (Trials) TL } & \text { Trials } & 1.3,12.9 & 51.2 & <0.001 & 2.4,23.5 & 11.4 & <0.001\end{array}$

P3b

This article is protected by copyright. All rights reserved. 


\begin{tabular}{llllllll} 
& & df & F & p & df & F ex. 1 & p ex. 1 \\
\hline 2-way ANOVA & Condition & 1,10 & 35.5 & $<0.001$ & 1,10 & 31.6 & $<0.001$ \\
& Trial & $7.2,72.1$ & 6.1 & $<0.001$ & $7,70.3$ & 2.8 & $<0.05$ \\
& & & & & & & \\
& Cond. ${ }^{*}$ Trial & $6.1,61.2$ & 7.7 & $<0.001$ & $5.7,56.8$ & 4 & $<0.005$
\end{tabular}

Planned Comp.

$\begin{array}{llllllll}\text { (Trials) TC } & \text { Trials } & 5.9,59.4 & 0.7 & 0.63 & 5.9,59.4 & 0.7 & 0.85 \\ \text { (Trials) TL } & \text { Trials } & 6.4,63.8 & 12.5 & <0.001 & 6.2,62.1 & 5.9 & <0.001\end{array}$

Alpha Power

\begin{tabular}{lllllllll} 
& & df & F & p & df & F ex. 1 & p ex. 1 \\
\hline 2-way ANOVA & Condition & 1,10 & 5.1 & $<0.05$ & 1,10 & 5.2 & $<0.05$ \\
& Trial & $4.7,47.2$ & 7.5 & $<0.001$ & $4.9,48.9$ & 6.2 & $<0.001$ \\
& Cond. ${ }^{*}$ Trial & $5.5,54.6$ & 1.5 & 0.187 & $5.4,53.9$ & 1.5 & 0.187
\end{tabular}

Planned Comp.

$\begin{array}{llllllll}\text { (Trials) TC } & \text { Trials } & 5.1,51.3 & 4.3 & <0.05 & 5,50 & 2.9 & <0.05 \\ \text { (Trials) TL } & \text { Trials } & 4.8,47.8 & 5.6 & <0.001 & 4.9,49.2 & 5.1 & <0.001\end{array}$

This article is protected by copyright. All rights reserved. 
Table 2. A summary of correlation analysis results for Target Learning (TL) and Target Cued (TC) experimental conditions. Analysis was carried out for both experimental conditions with inclusion and exclusion of the first trial. Correlations between movement time (MT) and P3b amplitude, MT and alpha power and P3b amplitude and alpha power are included. R- and p-values are reported. $(* \mathrm{p}<$ $0.015, * * \mathrm{p}<0.005, * * * \mathrm{p}<0.001)$.

\begin{tabular}{|c|c|c|c|c|}
\hline & $\mathrm{TL}$ & $\mathrm{TC}$ & TL ex. $1^{\text {st }}$ trial & TC ex. $1^{\text {st }}$ trial \\
\hline \multirow{3}{*}{ MT vs. P3b } & $R=0.85$ & $R=0.04$ & $R=0.95$ & $R=0.29$ \\
\hline & & & & \\
\hline & $\mathrm{p}<.001 * * *$ & $p=0.828$ & $\mathrm{p}<.001 * * *$ & $p=0.14$ \\
\hline \multirow{3}{*}{ MT vs. alpha } & $R=-0.47$ & $R=-0.45$ & $R=-0.63$ & $R=0.015$ \\
\hline & & & & \\
\hline & $p<.015 *$ & $p=0.14$ & $\mathrm{p}<.001 * * *$ & $p=0.937$ \\
\hline \multirow{3}{*}{ P3b vs. alpha } & $R=-0.64$ & $R=0.27$ & $R=-0.60$ & $R=0.197$ \\
\hline & & & & \\
\hline & $\mathrm{p}<.001 * * *$ & $p=0.15$ & $\mathrm{p}<.001 * * *$ & $p=0.305$ \\
\hline
\end{tabular}

This article is protected by copyright. All rights reserved. 
Table 3. A summary of the single participant reproducibility analysis for Target Learning (TL) and Target Cued (TC) conditions. Significance has been defined as a p-value less than 0.015 . The number of participants with significant and non-significant correlations between movement time (MT) and P3b amplitude, MT and alpha power and P3b amplitude and alpha power are reported.

\begin{tabular}{|c|c|c|}
\hline & $\begin{array}{l}\text { Significant } \\
(p<0.015)\end{array}$ & Non-significant \\
\hline TL: MT vs. P3b & 8 & 3 \\
\hline TL: MT vs. alpha & 6 & 5 \\
\hline TL: P3b vs. alpha & 2 & 9 \\
\hline TC: MT vs. P3b & 0 & 11 \\
\hline TC: MT vs. alpha & 1 & 10 \\
\hline TC: P3b vs. alpha & 1 & 10 \\
\hline
\end{tabular}

This article is protected by copyright. All rights reserved. 

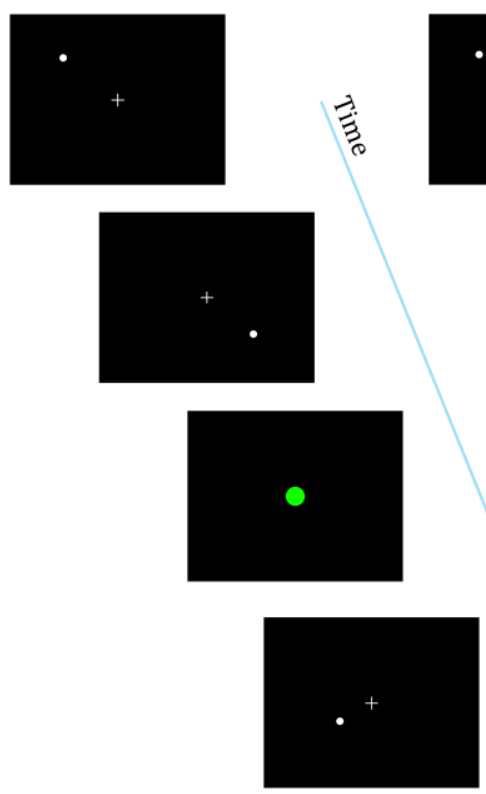

b

Representative Trajectories: TL

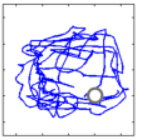

trial 1
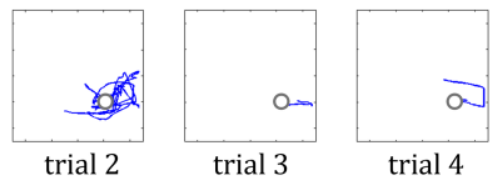

O

$+$
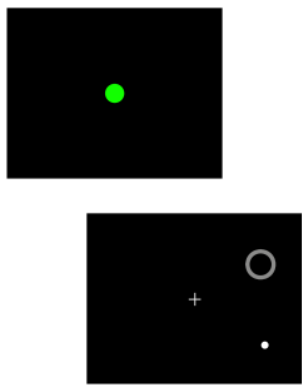

Visuospatial search

Duration $=$ Movement Time $(M T)$

Target location

Feedback stimulus

Duration $=500 \mathrm{~ms}$

Select start position for next trial

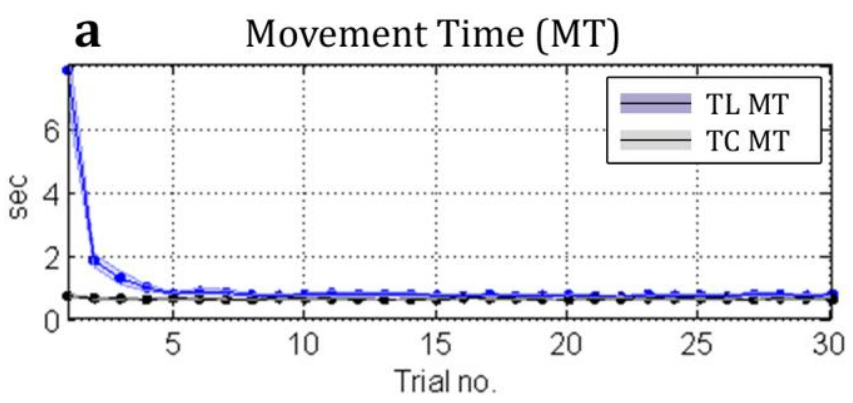

\section{b Movement Time (MT): excluding trial 1}

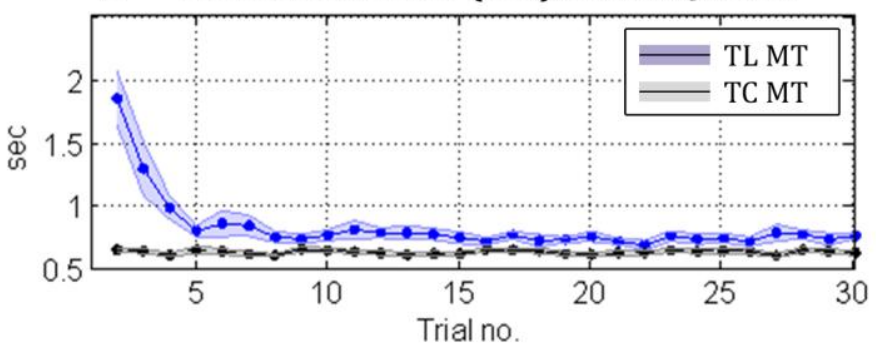

This article is protected by copyright. All rights reserved. 

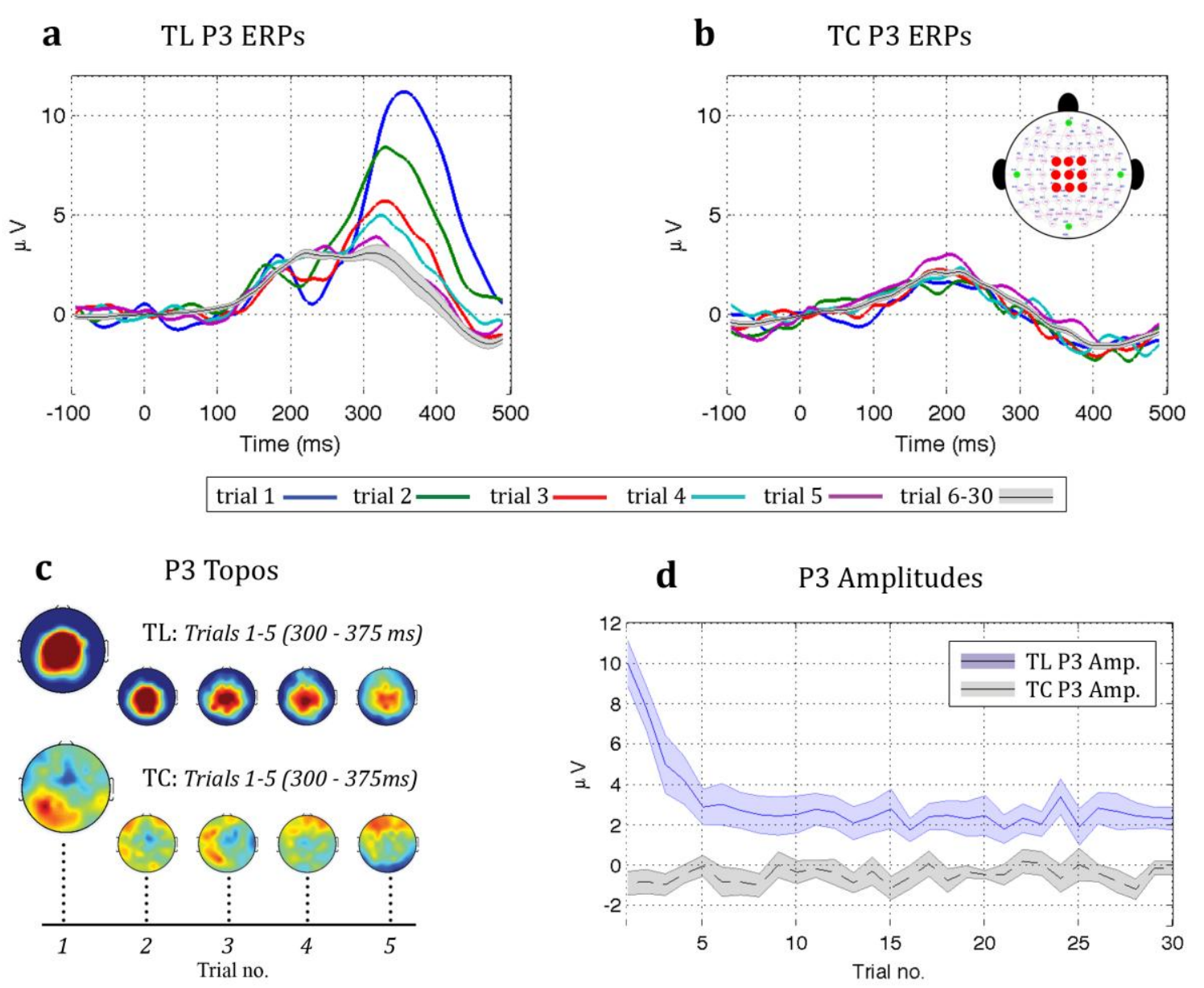

This article is protected by copyright. All rights reserved. 
a

TL Alpha Power
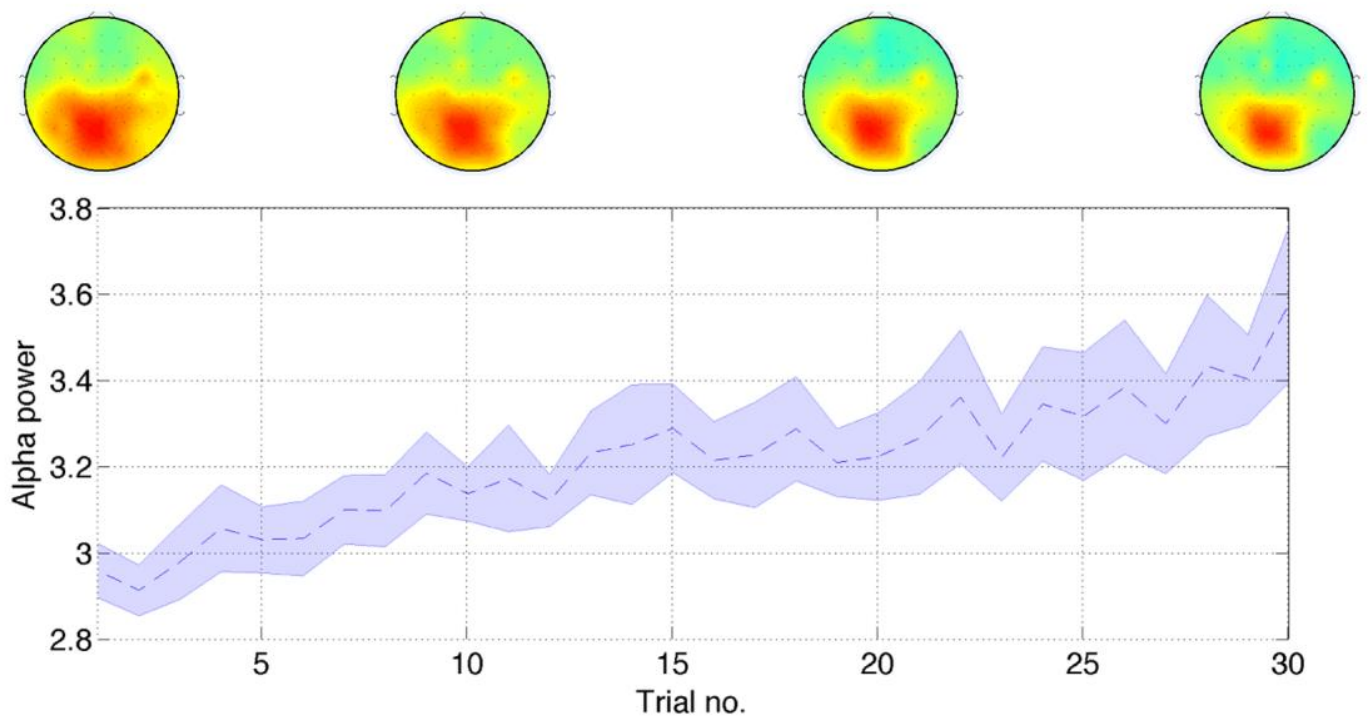

b

TC Alpha Power
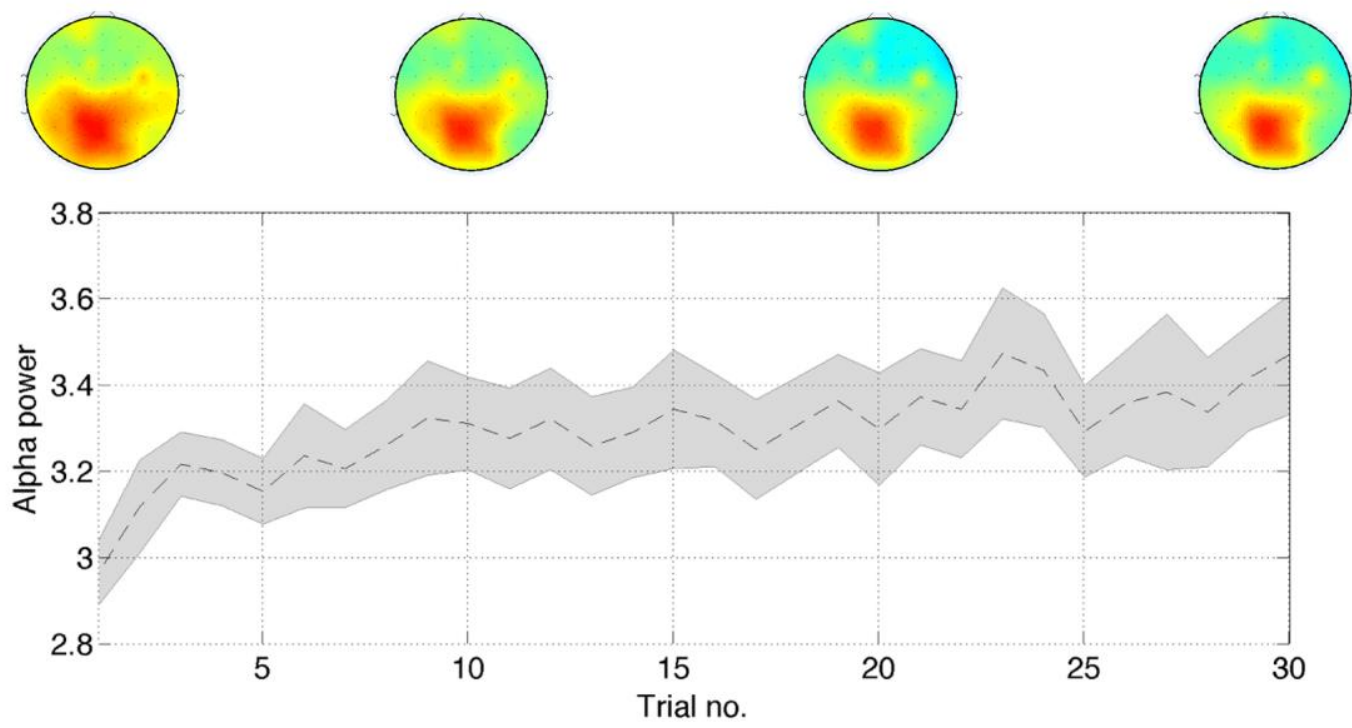

TL alpha $=$ TC alpha

This article is protected by copyright. All rights reserved. 
a MT vs. P3 MT vs. alpha P3 vs. alpha

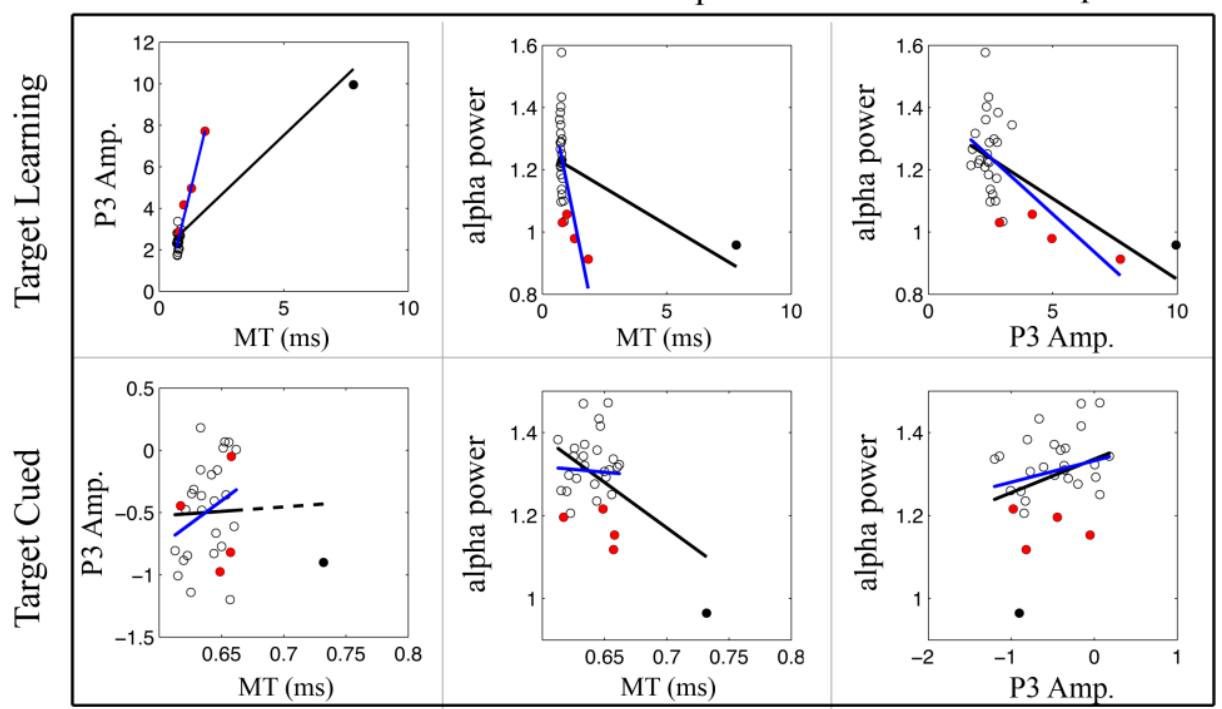

Ex. 1st trial, $\mathrm{p}<.015$

All points, $\mathrm{p}<.015$

- - Ex. 1st trial, $\mathrm{p}>.015$

- - All points, $p>.015$

- Trial 1

- Trial 2-5

○ Trial 6-30

This article is protected by copyright. All rights reserved. 